\title{
Impacto de la información disonante y consistente sobre la campaña Marca Perú en la identidad nacional y el bienestar social*
}

\author{
Impact of Dissonant Information about the Peruvian Brand \\ Campaign on National Identity and Social Well-Being
}

Recibido: octubre 10 de 2013 | Revisado: agosto 14 de 2014 | Aceptado: agosto 14 de 2014

\author{
MATHIAS SCHMITZ** \\ Pontificia Universidad Católica del Perú, Perú \\ Agustín EsPINOSA *** \\ Pontificia Universidad Católica del Perú, Perú
}

doi:10.11144/Javeriana.upsy13-5.iidc

Para citar este artículo: Schmitz, M. \& Espinosa, A. (2015). Impacto de la información disonante y consistente sobre la campaña Marca Perú en la identidad nacional y el bienestar social. Universitas Psychologica, 14(1), 329-338. http://dx.doi. org/10.11144/Javeriana.upsy13-5.iidc

* Artículo original resultado de investigación

*** Licenciado en Psicología Social de la Pontificia Universidad Católica del Perú (PUCP). Predocente del Departamento de Psicología de la PUCP. Miembro del Grupo de Psicología política de la PUCP. Correo electrónico: mathias.schmitz@pucp.pe

**** Doctor en Psicología Social de la Universidad del País Vasco (UPV/EHU) y Licenciado en Psicología por la Pontificia Universidad Católica del Perú (PUCP). Profesor Ordinario del Departamento de Psicología de la PUCP. Coordinador del Grupo de Psicología Política de la PUCP. Correo electrónico: agustin.espinosa@pucp.edu.pe

\section{RESUMEN}

El impacto de la información disonante y consistente sobre la campaña de la marca país en la identidad nacional y el bienestar social es estudiado mediante un diseño experimental mixto a partir de una muestra de estudiantes de una universidad de Lima metropolitana $(N=102)$. Los resultados muestran que la exposición a información negativa sobre un comercial de tipo "Marca País" ejerce una influencia negativa sobre los componentes de la identidad nacional y el bienestar social, mientras que la información positiva produce el efecto inverso. Estos resultados ponen en evidencia la importancia que tiene el manejo adecuado y responsable de los contenidos asociados a las campañas que buscan exaltar el endogrupo nacional, así como de los discursos críticos que las acompañan.

Palabras clave

identidad nacional; bienestar social; marca país

\footnotetext{
A B S T R A C T

The impact of dissonant and consonant information about the national brand campaign on national identity and social welfare is studied using a mixed experimental design from a sample of students from a university of Lima $(N=102)$. The results show that exposure to negative information about a commercial related to the National Brand exert a negative influence on the components of national identity and social wellbeing, while positive information produces the inverse. These results highlight the importance of proper and responsible handling for the content associated with campaigns that seek to exalt the national ingroup and critical discourses that accompany them.

Keywords

national identity; social wellbeing; national brand
} 


\section{Identidad social y bienestar social}

La identificación colectiva constituye un proceso psicológico universal y adaptativo que ha contribuido a la resolución de problemas de supervivencia a los cuales ha estado expuesta la especie humana a lo largo de su historia evolutiva (Hogg \& Abrams, 1988). Por ello, existe una tendencia natural en las personas de clasificarse a sí mismas y a otros sobre la base de categorías sociales (Tajfel \& Turner, 2004). La pertenencia a estas agrupaciones permite al individuo desarrollar su identidad social, entendida como "[...] aquella parte del autoconcepto de un individuo que deriva del conocimiento de su pertenencia a un grupo o grupos sociales, junto con el significado valorativo y emocional asociado a dicha pertenencia” (Tajfel, 1984, p.292).

La construcción y mantenimiento de la identidad social se ve mediada por un conjunto de motivos identitarios, los cuales orientan a las personas hacia determinados estados identitarios y los repelen de otros. Estos motivos son: autoestima, continuidad, pertenencia, autoeficacia, comprensión y significado (Vignoles, Regalia, Manzi, Golledge, \& Scabini, 2006). Aquellos aspectos de las identidades actuales que mejor satisfacen los motivos identitarios son asociados con la centralidad percibida y son enfatizados en la auto-presentación (Vignoles, et al., 2006). Uno de los motivos más importantes es el de la autoestima, el cual refiere a la motivación de reforzar y conservar una concepción positiva de sí mismo (Gecas, 1982) y que en su dimensión colectiva comprende la actitud del individuo hacia las categorías y grupos sociales a los que pertenece (Luhtanen \& Crocker, 1992; Crocker \& Major, 1989).

Por otra parte, el bienestar social se refiere a "[...] la valoración que hacemos de las circunstancias y el funcionamiento dentro de la sociedad" (Keyes, 1998, p. 122). El bienestar social está estructurado en cinco dimensiones: 1) la integración social, referida al sentido de pertenencia e integración con respecto a la sociedad; 2) la aceptación social, la cual refleja una percepción positiva de los miembros de la comunidad; 3) la contribución social, asociada al sentimiento de verse a sí mismo como una persona útil y que contribuye con la so- ciedad; 4) la actualización social, referida a la visión de la sociedad como potencialmente positiva para el crecimiento personal; y 5) la coherencia social, asociada a la visión de la sociedad como comprensible y con sentido (Keyes, 1998).

Existen argumentaciones conceptuales y evidencia empírica que respaldan la idea sobre el efecto positivo que ejerce la identidad social en el bienestar (Espinosa \& Tapia, 2011; Lyubomirsky, Tkach, \& DiMatteo, 2006). Al respecto, se ha sugerido que una mayor identificación con un grupo promueve el bienestar (Jetten, Branscombe, Schmitt, \& Spears, 2001) y que aquellos eventos relevantes para la identidad afectarían al mismo (Burke, 1991). En concordancia con estos hallazgos, se encontró que la expresión positiva de la identidad nacional favorece el bienestar social en Perú y otros países de América Latina (Espinosa, Beramendi, \& Zubieta, 2013; Espinosa, Schmitz, \& Rottenbacher, 2013; Espinosa \& Tapia, 2011; Laca, Mejía, \& Yañez, 2010).

\section{Identidad nacional e identidad nacional peruana}

La identidad nacional es un subtipo específico de identidad social en la medida que hace referencia a la nación como categoría social (Nigbur \& Crinnirella, 2007). El tema de la identidad nacional peruana cobra especial relevancia al considerar la trágica historia que ha tenido el país, así como sus connotaciones y atributos no siempre positivos (Espinosa, 2011). Debido al bajo estatus adscrito al país y a una comparación social desventajosa con naciones de mayor estatus, se ha formulado la hipótesis de la prevalencia de una identidad nacional frágil y negativa (Espinosa, 2003; 2011; Rottenbacher \& Espinosa, 2010; Salgado, 1999). Esta última trae consigo una serie de consecuencias negativas para el individuo, puesto que entre otras cosas, representa un riesgo potencial para la autoestima colectiva (Tajfel \& Turner, 1986), refuerza autoestereotipos negativos del endogrupo (Tajfel, 1984) y fomenta una visión pesimista sobre el futuro del mismo (Montero, 1996).

Sin embargo, a pesar de la hipótesis propuesta, los resultados de investigaciones recientes sobre 
identidad nacional en Perú sugieren niveles relativamente altos de identificación con el país y una valoración positiva de este, junto con auto-estereotipos ambivalentes, mas no negativos en sentido estricto (Espinosa, 2011; Salgado, 1999). Espinosa (2011) plantea que el contexto social peruano juega un papel importante en la constitución de la identidad nacional en el país. Así, la pertenencia e identificación con diferentes grupos étnicos y sociales que conforman el mismo, junto a los diversos estereotipos y valoraciones de estos grupos, condicionarían las interpretaciones de la categoría nacional, dando lugar a diferentes perspectivas de lo que es ser peruano.

\section{La campaña Marca Perú y la representación positiva del endogrupo nacional}

La Marca Perú es una marca país, la cual es un símbolo que identifica un país y lo distingue de los demás. Esta pone en relieve las cualidades positivas, memorables, atractivas e únicas de la nación, promoviendo y defendiendo así su reputación en el plano internacional y nacional. Se espera que las campañas asociadas a la marca país permitan atraer inversiones extranjeras y promuevan las exportaciones y el turismo. Además, al valorizar los aspectos positivos de la nación, también se espera que genere un impacto positivo en el orgullo nacional, la confianza y la coherencia interna percibida (Perú Info, s.f.).

En este sentido, el comercial de "Perú, Nebraska”, presentado en mayo del 2011 como parte de la campaña Marca Perú, tuvo como objetivo difundir la Marca Perú a los peruanos dentro y fuera del país (Perú Info, s.f.). Este comercial muestra un pueblo homónimo "Peru" en Nebraska (Estados Unidos) en el cual aparecen celebridades peruanas que promueven los atributos positivos asociados al país como por ejemplo, el arte culinario, la cultura peruana o los deportes en los que se destacan. Dicho comercial ha tenido un alto nivel de difusión y recordación entre los peruanos (Ipsos Apoyo, 2012).

Empero, la campaña de Marca Perú también ha generado una ola de críticas, las cuales han tenido una amplia difusión a través de blogs, artículos de periódicos y redes sociales (Faverón, 2011; Perú21, 2012; Peruanista, 2011; Tafur, 2011). Una de las críticas que aparece con mayor frecuencia es que, en lugar de promocionar un país multicultural, lo que se ve reflejado en el comercial es el Perú visto desde los sectores de poder de Lima; bajo el argumento de que la mayoría de actores que figura en el comercial son blancos y aquellos con rasgos indígenas y afroperuanos son cantantes y bailarines que cumplen un papel secundario.

\section{Información disonante sobre el endogrupo nacional}

Las personas que se identifican con un grupo social están motivadas a mantener un concepto positivo del grupo con el que se identifican (Tajfel \& Turner, 1986). Así, la información negativa o disonante sobre el endogrupo nacional representaría una amenaza directa para la identidad social de la persona y su autoestima. Por ello, cuando un sujeto se ve expuesto o posee dos o más cogniciones (e.g. actitudes, deseos, creencias) relevantes pero inconsistentes entre sí -por ejemplo, información favorable y desfavorable sobre el endogrupo nacional-, se produce una disonancia cognitiva. Esta incompatibilidad genera un estado de inconformidad psicológica que el individuo busca eliminar o reducir (Festinger, 1964).

Específicamente, cuando la información disonante representa una amenaza para el concepto que se tiene del endogrupo, las personas utilizan diversas estrategias de afrontamiento como puede ser el favoritismo endogrupal, expresado como la tendencia de los individuos a favorecer o valorar positivamente su grupo (Brewer, 1999). Al respecto se encontró que las personas con una identificación nacional más alta tienden a hacer un mayor uso del mecanismo de afrontamiento cuando se ven enfrentados a información negativa sobre el endogrupo nacional (Rensman, 2004).

La relevancia de este estudio se evidencia al tomar en cuenta la necesidad de reconstruir y forjar una identidad nacional sobre la base de una autoestima y auto estereotipos colectivos positivos e inclusivos (Espinosa, 2003; 2011; Genna, Espi- 
nosa, \& Páez, 2010). En este sentido, discursos de comunicación como las campañas de marca país, las cuales representan aproximaciones sociales que buscan exaltar el endogrupo nacional, constituyen un intento significativo por responder a estas necesidades. Sin embargo, estas campañas también generan una ola de críticas que se difunden ampliamente en diversos medios formales e informales. Esta incompatibilidad entre discursos y/o creencias sobre el endogrupo nacional podría generar disonancia cognitiva en las audiencias, pudiendo repercutir negativamente en la eficacia de la campaña, así como en la identificación con el endogrupo nacional y el bienestar social.

Sobre esta base, el presente estudio, de índole experimental, tiene como objetivo principal analizar el impacto de la estrategia comunicacional "Peru Nebraska" en la identidad nacional y el bienestar social, cuando viene acompañada de información positiva o negativa sobre la misma. Se plantean las siguientes hipótesis:

- (H1) Los participantes expuestos a la información disonante (comunicación más información negativa) reportarán menores niveles de identificación nacional, autoestima colectiva, menor acuerdo con contenidos estereotípicos positivos y mayor acuerdo con estereotipos negativos que los participantes expuestos a la información consistente (comunicación más información positiva).

- (H2) Los participantes expuestos a la información disonante reportarán puntuaciones menores en bienestar social que los participantes expuestos a la información consistente.

\section{Método}

\section{Participantes}

Los participantes fueron estudiantes universitarios de una universidad privada de Lima metropolitana $(N=102)$. La mayoría de la muestra estuvo compuesta por mujeres $(62.70 \%)$. Todos fueron peruanos y/o residentes en el Perú, con edades entre los 16 y 25 años $(M=18.60, D E=1.93)$. La distribución por nivel socioeconómico (NSE) fue la siguiente: $35.30 \%$ NSE alto, $42.20 \%$ NSE medio alto, $18.60 \%$
NSE medio y $2.00 \%$ NSE medio bajo. Asimismo $81.40 \%$ de participantes habían nacido en Lima y $86.30 \%$ había visto el comercial de Marca Perú previamente al estudio.

Los participantes fueron asignados aleatoriamente a la condición de información consistente ( $n$ $=51)$ o la condición de información disonante $(n$ $=51$ ). Se controló la homogeneidad de los grupos experimentales en las variables: edad, sexo, NSE, lugar de nacimiento y si habían visto o no el video antes de participar en el estudio. A pesar de la aleatorización, los grupos experimentales difirieron significativamente respecto a la variable sexo: el porcentaje de mujeres en la condición consistente (74.50\%) fue significativamente mayor que en la condición disonante (51.00\%), $\chi^{2}(1)=6.04, p<0$ .05. Sin embargo, se estima que dicha diferencia no afectó el resultado de los análisis ya que el experimento no estuvo estructurado en base a esa variable y el tamaño del efecto de la referida diferencia fue pequeño, $\phi=-.24, p=<0.05$.

Se tuvieron las consideraciones éticas sobre la participación a través del consentimiento informado.

\section{Estímulos materiales}

Ficha de datos. Se creó una ficha de datos para recoger información sobre la edad, sexo, nivel socio económico (NSE) y lugar de nacimiento. También se preguntó a los participantes si habían visto previamente el video "Peru, Nebraska" utilizado en la evaluación.

Nivel de identificación con el Perú. Esta medida consta de un ítem que plantea la siguiente pregunta: ¿Cuál es su grado de identificación con el Perú? Los valores de respuesta de esta escala van del 1 (nada) al 5 (total).

Escala de autoestima colectiva peruana. Se utilizó la versión adaptada a la categoría social peruana por Espinosa (2011) de la sub-escala de autoestima colectiva privada de Luhtanen y Crocker (1992). La escala está compuesta por cuatro ítems que evalúan la relación afectiva de los participantes en el estudio tomando en cuenta su identificación con el Perú. Las respuestas van del 1 (totalmente en desacuerdo) 
al 5 (totalmente de acuerdo). La escala total obtuvo una confiabilidad aceptable $(\alpha=0.77)$ de acuerdo a los criterios establecidos por Mezulis, Abramson, Hyde y Hankin (2004).

Escala de autoestereotipo colectivo peruano. Esta escala fue construida por Espinosa (2003) y está compuesta por 24 adjetivos que describen a los peruanos. Cada adjetivo recibe una puntuación por los participantes que va desde 1 (totalmente en desacuerdo) hasta 5 (totalmente de acuerdo). Los 24 ítems están agrupados en cuatro dimensiones (Espinosa, 2011), las cuales obtuvieron niveles de confiabilidad aceptables: peruanos desconfiables $(\alpha$ $=.079)$, peruanos confiables $(\alpha=0.79)$, peruanos capaces y alegres $(\alpha=0.55)$ y peruanos patriotas y solidarios $(\alpha=0.58)$.

Escala de bienestar social. Se utilizó la versión de Blanco y Díaz (2005) de la Escala de Bienestar Social de Keyes (1998), la cual consta de quince ítems que conforman cinco dimensiones. Cada ítem debe ser valorado en una escala que va del 1 (totalmente en desacuerdo) hasta el 5 (totalmente de acuerdo). Los quince ítems conforman cinco dimensiones con coeficientes de confiabilidad aceptables: integración social $(\alpha=0.55)$, aceptación social $(\alpha=$ 0.69), contribución social $(\alpha=0.75)$, actualización social $(\alpha=0.50)$ y coherencia social $(\alpha=0.55)$. Asimismo la escala total obtuvo una confiabilidad aceptable $(\alpha=0.77)$.

Versión corta del comercial de la campaña Marca Perú: "Perú, Nebraska". Se creó una versión corta del comercial "Peru, Nebraska" presentado como parte de la campaña Marca Perú. El comercial original tenía una duración de quince minutos y fue editado por los investigadores a una versión de siete minutos, seleccionando aquellos fragmentos que dan cuenta de los impactos positivos en materia de fenómenos sociales: diversidad cultural, gastronomía, deporte, música, bailes típicos, entre otros.

Textos con información positiva o negativa sobre el comercial de Marca Perú. Se crearon dos textos a partir de la información recopilada en blogs y periódicos. Uno de ellos con información positiva y otro con información negativa sobre el video "Peru, Nebraska" para elaborar las condiciones consistente y disonante, respectivamente.

\section{Procedimiento}

Se utilizó un diseño experimental mixto con dos grupos experimentales (condición disonante y condición consistente) y medidas repetidas (grado de identificación nacional y la escala de autoestima colectiva peruana). Además de ello, también se midieron otras variables dependientes que resultaron de interés para el presente estudio.

La aplicación del estudio se realizó de manera individual. Los participantes fueron convocados de manera intencional en un salón de la universidad. Después de haber firmado el consentimiento informado, se les pidió llenar la primera parte del cuestionario, la cual comprendía: ficha de datos, nivel de identificación con el Perú y escala de autoestima colectiva peruana. A continuación se proyectó el video "Peru, Nebraska" y se solicitó a los participantes estar atentos al mismo. Después de la proyección se repartió de manera aleatoria entre los participantes un texto con información positiva o negativa sobre el video presentado. De los 102 participantes, 51 recibieron un texto con información consistente (video más información positiva) y 51 con información disonante (video más información negativa). Se pidió a los participantes leer atentamente esa información. Después de haber leído el texto, se procedió con la aplicación de la segunda parte del cuestionario. Esta segunda parte comprendía: el nivel de identificación con el Perú, la escala de autoestima colectiva peruana, la escala de auto-estereotipos y la escala de bienestar social. Luego de haber finalizado el cuestionario se agradeció a cada persona por su participación. Los datos recopilados en este estudio fueron procesados con el software estadístico IBM SPSS Statistics v21.

\section{Resultados}

\section{Grado de Identificación con el Perú}

Se examinaron las diferencias en el grado de identificación nacional pre-test entre los grupos. El grado de identificación con el Perú pre-test fue significativamente más alto en el grupo consis- 
TABLA 1

Medias con ajuste y sin ajuste de la identificación con el Perú post-test con la identificación con el Perú-pre-test como covariable

\begin{tabular}{llllll}
\hline & & \multicolumn{2}{c}{ Sin ajuste } & \multicolumn{3}{c}{ Con ajuste } \\
\cline { 3 - 7 } Condición & $N$ & $M$ & $D E$ & $M$ & $D E$ \\
Consistente & 51 & 4.14 & 0.63 & 4.02 & 0.06 \\
Disonante & 51 & 3.70 & 0.68 & 3.82 & 0.06 \\
\hline
\end{tabular}

Fuente: elaboración propia

tente, $M=4.02, D E=0.65$, en comparación al disonante $M=3.68, S D=0.74, t(96.74)=2.45$, $p<0.05, d=0.49$.

Debido a la diferencia inicial entre grupos respecto a la identificación con el Perú en el pre-test, se realizó un ANCOVA para determinar el efecto de la información consistente y disonante en la identificación con el Perú en el post-test. Se encontraron diferencias significativas respecto a esta variable entre las condiciones experimentales después de ajustar los niveles de identificación con el Perú pretest, $F(1,98)=4.88, p<0.05, \eta^{2}=0.05$. Se realizó un análisis post-hoc con el ajuste de Bonferroni, resultando que la identificación con el Perú en el post-test era significativamente mayor en la condición consistente que en la condición disonante, $p$ $<0.05, d=1.67$ (ver Tabla 1).

A continuación se realizó una comparación de medias para muestras relacionadas en cada condición. No se reportaron diferencias significativas entre el pre-test, $M=4.02, D E=0.65$ y el post-test, $M=4.14, D E=0.63$ en la condición consistente, $t(50)=-1.77, p=0.08, d=0.18$. Del mismo modo, tampoco se encontraron diferencias en la condición disonante entre el pre-test, $\mathrm{M}=3.68, \mathrm{DE}=0.74$, y el post-test, $M=3.70, D E=0.68, t(49)=-0.30$, $p=0.77, d=0.03$.

\section{Autoestima colectiva}

Los datos fueron analizados usando un ANOVA mixto con el tiempo de medida (pre-test, post-test) como factor intra-sujeto y la condición experimental (consistente, disonante) como factor entre-sujeto. Se reportó una interacción significativa entre el tiempo y las condiciones experimentales, $F(1,97)=$ $13.65, p<.01, \eta^{2}$ parcial $=0.12$, junto a un efecto principal del factor grupo, $F(1,97)=5.88, p<$ $0.05, \eta^{2}$ parcial $=0.06$. El efecto principal del factor tiempo no resultó significativo, $F(1,97)=0.07, p=$ $0.79, \eta^{2}$ parcial $<0.01$.

A continuación se examinaron los efectos simples en cada factor. A nivel entre-sujeto, no se reportaron diferencias significativas entre la autoestima colectiva pre-test entre la condición consistente, $M$ $=4.39, \mathrm{DE}=0.66$ y la condición disonante, $\mathrm{M}=$ $4.24, D E=0.67, t(98)=1.10, p=0.28, d=0.23$. Sin embargo, la autoestima colectiva post-test fue significativamente más alta en el grupo expuesto a la condición consistente, $M=4.54, D E=0.61$

TABLA 3

Comparación de medias para los autoestereotipos por condición

\begin{tabular}{|c|c|c|c|c|c|c|c|c|}
\hline \multirow[b]{2}{*}{ Variable } & \multicolumn{2}{|c|}{ Consistente } & \multicolumn{2}{|c|}{ Disonante } & \multirow{2}{*}{ 95\% IC dif. de medias } & \multirow[b]{2}{*}{$T$} & \multirow[b]{2}{*}{$d$} & \multirow[b]{2}{*}{$g l$} \\
\hline & $n$ & $M(D E)$ & $n$ & $M(D E)$ & & & & \\
\hline Desconfiables & 50 & $2.70(.67)$ & 50 & $3.03(.58)$ & {$[-.57,-.07]$} & $-2.57 *$ & 0.53 & 98 \\
\hline Confiables & 50 & $3.17(.55)$ & 50 & $2.92(.41)$ & {$[.05, .44]$} & $2.49 *$ & 0.52 & 98 \\
\hline Capaces y alegres & 51 & $4.44(.47)$ & 51 & $4.20(.44)$ & {$[.06, .41]$} & $2.62 *$ & 0.53 & 100 \\
\hline Patriotas y solidarios & 50 & $3.56(.69)$ & 51 & $3.34(.72)$ & {$[-.07, .49]$} & 1.52 & 0.31 & 99 \\
\hline
\end{tabular}

$*_{p}<.05, * * p<.01$

Fuente: elaboración propia 
a comparación del grupo expuesto a la condición disonante, $M=4.06, D E=0.76, t(92)=3.42, p$ $<0.01, d=0.70$. A nivel intra-sujeto, la autoestima colectiva pre-test, $M=4.39, \mathrm{DE}=0.66$ fue significativamente más baja que la autoestima colectiva post-test, $M=4.54, D E=0.61$ en el grupo expuesto a la información consistente, $t(49)=-2.71, p<$ $0.01, d=0.24$.

En la condición disonante, la autoestima colectiva pre-test, $M=4.23, D E=0.67$ fue significativamente más alta que la autoestima colectiva post-test, $M=4.06, D E=0.76, t(48)=2.55, p<$ $0.05, d=0.24$.

\section{Autoestereotipos nacionales}

En una escala del uno al cinco, se comparó en la aplicación post-test el grado de acuerdo respecto a los estereotipos que describen a los peruanos en general. Los resultados se muestran en la tabla a continuación:

Como se puede apreciar en la Tabla 3, en la condición consistente, los peruanos son percibidos como más confiables, capaces y alegres y menos desconfiables que en la condición disonante. No se encontraron diferencias significativas respecto al contenido auto-estereotípico de peruanos patriotas y solidarios.

\section{Bienestar Social}

En una escala del uno al cinco, se compararon los niveles de bienestar social entre los dos grupos. Los resultados se presentan en la siguiente tabla:

TABLA 4

Comparación de medias en bienestar social por condición
Se aprecia en la Tabla 4 que las dimensiones de aceptación y actualización, así como el bienestar social en general, fueron significativamente más altas en la condición consistente que en la disonante.

\section{Discusión}

Impacto de la información disonante/ consistente en la identidad nacional

La primera hipótesis (H1) de este estudio se ve comprobada al observar que los componentes de la identidad nacional (grado de identificación, autoestima colectiva y auto estereotipos nacionales) se ven afectados positivamente cuando los sujetos son expuestos a una pauta de comunicación positiva sobre el endo-grupo nacional con información positiva que la acompaña, mientras que se produce el efecto contrario con la información disonante.

En primer lugar no se reportaron diferencias significativas con respecto al grado de identificación con el Perú a nivel intra-sujeto, lo cual supone que dicha variable constituye un rasgo relativamente estable a lo largo del tiempo y es poco vulnerable a la influencia externa. Sin embargo, la intervención experimental sí generó cambios significativos a nivel inter-sujeto, siendo esta más baja en el caso del grupo expuesto a información crítica sobre el endogrupo nacional. Este último resultado es coherente con la idea de que una de las estrategias adoptadas por los individuos, en un intento por proteger su propio auto-concepto y autoestima vinculados a

\begin{tabular}{|c|c|c|c|c|c|c|c|c|}
\hline \multirow{2}{*}{ Variable } & \multicolumn{2}{|c|}{ Consistente } & \multicolumn{2}{|c|}{ Disonante } & \multirow{2}{*}{ 95\% IC dif. de medias } & & \multirow[b]{2}{*}{$a$} & \multirow[b]{2}{*}{$g l$} \\
\hline & $n$ & $M(D E)$ & $n$ & $M(D E)$ & & & & \\
\hline Integración social & 51 & $4.04(.65)$ & 51 & $3.80(.63)$ & {$[-.01, .49]$} & 1.91 & 0.37 & 100 \\
\hline Aceptación social & 50 & $3.50(.74)$ & 51 & $3.18(.75)$ & {$[.02, .61]$} & $2.15 *$ & 0.43 & 99 \\
\hline Contribución social & 51 & $4.60(.52)$ & 51 & $4.46(.59)$ & {$[-.08, .35]$} & 2.26 & 0.25 & 100 \\
\hline Actualización social & 50 & $4.38(.59)$ & 50 & $4.04(.77)$ & {$[.07, .61]$} & $2.48 *$ & 0.50 & 98 \\
\hline Coherencia social & 51 & $4.52(.69)$ & 51 & $4.27(.67)$ & {$[-.02, .51]$} & 1.86 & 0.37 & 100 \\
\hline Bienestar social total & 49 & $4.42(.37)$ & 50 & $3.94(.48)$ & {$[.11, .45]$} & $3.26 * *$ & 1.12 & 97 \\
\hline
\end{tabular}

$* p<.05, * * p<0.01$

Fuente: elaboración propia 
su identidad social, es reducir el grado de identificación con dicho grupo (Tajfel \& Turner, 1979).

En el caso de la autoestima colectiva, sí se reportaron diferencias significativas a nivel intra-sujeto entre las medidas pre y post test, siendo el post-test menor en el caso de la información disonante. Del mismo modo, también se corrobora el efecto de la intervención en el sentido esperado a nivel intersujeto. La relación entre las distintas medidas de la autoestima es más clara, puesto que es menos estable (Abrams \& Hogg, 1998; Gecas, 1982) y se vincula al carácter afectivo de la publicidad.

En la misma línea, se comprueba el efecto de la información disonante en la imagen de los peruanos al obtener niveles menores en dos de tres auto estereotipos nacionales con connotaciones positivas (peruanos confiables y peruanos capaces y alegres), así como mayores niveles de auto estereotipos nacionales con connotaciones negativas (peruanos desconfiables) en la condición disonante.

\section{Impacto de la información disonante/ consistente en el bienestar social}

Los resultados apoyan la segunda hipótesis de este estudio (H2) en la medida que los niveles de bienestar social total reportados fueron menores en la condición disonante y mayores en la consistente, lo cual advierte acerca del impacto que pueden tener los discursos críticos que acompañan las campañas marca país en el bienestar. En particular, se aprecia que esta discrepancia en los niveles de bienestar social está marcada sobre todo por diferencias en los niveles de actualización social y en menor medida, por los de aceptación social.

Se ha planteado que el bienestar social está en función de la valoración que hacen las personas de las circunstancias y el funcionamiento en la sociedad (Keyes, 1998). En este sentido, el discurso disonante exalta las condiciones negativas de la sociedad al poner al descubierto algunos de los problemas más graves que afectan al país, como la exclusión social, la desigualdad, la discriminación y la inseguridad ciudadana.

Tomando en cuenta los resultados previamente mencionados, es posible sugerir que se produjo una disonancia cognitiva en los sujetos de la condición expuestos a cogniciones relevantes pero inconsistentes entre sí respecto al endo-grupo nacional (condición disonante). Los menores niveles de bienestar social podrían interpretarse como el reflejo de la inconformidad y malestar psicológico producto de esta disonancia. Se puede especular que estos sujetos recurrieron al favoritismo endo-grupal como mecanismo para reducir la disonancia y mantener una imagen positiva del endo-grupo nacional. Sin embargo, la degradación de la identidad nacional en este grupo parece reflejar más bien un cambio de actitud a favor de las críticas, en la medida en que estas se encuentran ancladas en problemas sociales reales y tangibles. Esto último lleva a pensar que a pesar de que la identidad nacional se viene constituyendo actualmente de una manera menos negativa a lo que ocurría en décadas pasadas. El contraste con la realidad hace que esta sea poco estable y sensible a cambios drásticos ante información disonante compuesta por una comunicación que idealiza al país y la evidencia que niega esta idealización.

Finalmente, es posible concluir que los discursos críticos que acompañan a las campañas tipo marca país pueden tener un impacto significativo en los componentes de la identidad nacional y el bienestar y en términos generales, comprometer la eficacia de dichas campañas. Estos hallazgos ponen en evidencia la importancia de manejar adecuadamente el contenido y el mensaje asociado a las campañas orientadas a la promoción del endo-grupo nacional, así como el manejo de los discursos críticos que las acompañan.

\section{Referencias}

Abrams, D., \& Hogg, M. A. (1998). Comments on the motivational status of self esteem in social identity and intergroup discrimination. European Journal of Social Psychology, 18(4), 317-334.

Abrams, D., \& Hogg, M. A. (1990). An introduction to the social identity approach. En Abrams D., \& Hogg, M. A. (Eds.), Social identity theory: Constructive and critical advances (pp. 1-9). New York: Harvester Wheatsheaf. 
Blanco, A., \& Díaz, D. (2005). El bienestar social: Su concepto y medición. Psicothema, 17(4), 582-589.

Brewer, M. B. (1999). The Psychology of Prejudice: Ingroup Love or Outgroup Hate? Journal Of Social Issues, 55(3), 429.

Burke, P. J. (1991). Identity processes and social stress. American Sociological Review, 56(6), 836849. http://dx.doi.org/10.2307/2096259

Crocker, J., \& Major, B. (1989). Social stigma and selfesteem: The self-protective properties of stigma. Psychological Review, 96. 608-630. http://dx.doi. org/10.1037/0033-295X.96.4.608

Espinosa, A. (2003). Identidad social e identidad nacional en una muestra de triciclistas en Juliaca. Tesis de licenciatura no publicada. Pontificia Universidad Católica del Perú, Lima.

Espinosa, A. (2011). Estudios sobre identidad nacional en el Perú y sus correlatos psicológicos, sociales y culturales. (Tesis doctoral). Universidad del País Vasco-Euskal Herriko Unibertsitatea, País Vasco.

Espinosa, A., Beramendi, M., \& Zubieta, E. (2013). Identidad Nacional y Bienestar Social: una síntesis meta-analítica de estudios en Argentina, México y Perú. Manuscrito no publicado.

Espinosa, A., Schmitz, M., \& Rottenbacher, J.M. (2013). Ideological bases of national identity and their influence on subjective, psychological and social well-being in Peru. Manuscrito no publicado.

Espinosa, A., \& Tapia, G. (2011). Identidad nacional como fuente de bienestar subjetivo y social. Boletín de Psicología, 102, 71-87.

Faverón, G. (2011, 10 de mayo). "Marca Perú" o como tapar el sol con un dedo si el gobierno lo ordena. Puente Aéreo. Disponible en http://puenteareo1. blogspot.com/2011/05/marca-peru.html

Festinger, L. (1964). A theory of cognitive dissonance. Palo Alto, CA US: Stanford University Press.

Gecas, V. (1982). The self-concept. Annual Review of Sociology, 8, 1-33.

Genna, K., Espinosa, A., \& Páez, D. (2010). Cómo los vemos, cómo nos vemos. Influencia de la comparación social entre Perú y Chile en la identidad nacional peruana. Psicología Política, 41, 81-108.

Hogg, M.A., \& Abrams, D. (1988). Social identifications: A social psychology of intergroup relations and group processes. London: Routledge.
Ipsos Apoyo, Opinión y Mercado (2012). Evaluación de la imagen y campaña publicitaria de la Marca Perú. Disponible en http://www.ipsos-apoyo.com.pe Jetten, J., Branscombe, N. R., Schmitt, M. T., \& Spears, R. (2001). Rebels with a cause: Group identification as a response to perceived discrimination from the mainstream. Personality And Social Psychology Bulletin, 27(9), 1204-1213. http://dx.doi. org/10.1177/0146167201279012

Keyes, C. M. (1998). Social well-being. Social Psychology Quarterly, 61(2), 121-140. http://dx.doi. org/10.2307/2787065

Laca, F.A., Mejía, J.C., \& Yañez, C. (2010). Identidad mexicana e interés político: Predictores de bienestar social y anomía. Acta Universitaria, 20(2), 40-49.

Luhtanen, R., \& Crocker, J. (1992). A collective self-esteem scale: Self-evaluation of one's social identity. Personality And Social Psychology Bulletin, 18(3), 302-318. http://dx.doi. org/10.1177/0146167292183006

Lyubomirsky, S., Tkach, C., \& DiMatteo, M. M. (2006). What are the Differences between Happiness and Self-Esteem. Social Indicators Research, 78(3), 363 404. http://dx.doi.org/10.1007/s11205-005-0213-y

Mezulis, A. H., Abramson, L. Y., Hyde, J. S., \& Hankin, B. L. (2004). Is there a universal positivity bias in attributions? A meta-analytic review of individual, developmental and cultural differences in the selfserving attributional bias. Psychological Bulletin, 130(5), 711-747.

Montero, M. (1996). Identidad social negativa y crisis socioeconómica: Un estudio psicosocial. Revista Interamericana De Psicología, 30(1), 43-58.

Nigbur, D., \& Cinnirella, M. (2007). National identification, type and specificity of comparison and their effects on descriptions of national character. European Journal of Social Psychology, 37(4), 672-691. http://dx.doi.org/10.1002/ejsp.382.

Perú21 (2012, 19 de marzo). Iván Thays critica Marca Perú y patriotismo que solo celebra victorias. Disponible en http://www.rpp.com.pe/201203-19-ivan-thays-critica-marca-peru-y-patriotismo-que-solo-celebra-victorias-noticia_462796. html 
Perú Info (s.f.). ¿Qué es una "Marca País"?. Disponible en http://www.peru.info/\#what

Peruanista (2012, 5 de mayo). Marca Perú: campaña comercial diseñada por extranjeros para promocionar un país en venta. Disponible en http://peruanista. wordpress.com/2011/05/05/marca-peru-versioncomercial-de-la-peruanidad-con-acento-limeno/

Rensman, L. (2004). Collective Guilt, National Identity, and Political Processes in Contemporary Germany. En Branscombe, N. R., \& Doosje, B. (Eds.) Collective guilt: International perspectives (pp. 169-190). Cambrigde: Cambridge University Press.

Rottenbacher, J.M., \& Espinosa, A. (2010). Identidad nacional y memoria histórica colectiva en el Perú. Un estudio exploratorio. Revista de Psicología, 28(1), 147-174.

Salgado, C. (1999). ¿Quiénes somos los peruanos? Una perspectiva psicológica de la identidad nacional. Lima: Universidad de San Martín de Porres.

Tafur, N. (2011, 5 de septiembre). Marisol Espinoza critica a la Marca Perú. Diario Correo. Disponible en http:/diariocorreo.pe/ultimas/noticias/EPENSA-033726/marisol-espinoza-critica-a-la-marcaperu

Tajfel, H. (1984). Grupos humanos y categorías sociales: estudios de Psicología Social. Barcelona: Herder.
Tajfel, H., \& Forgas, J. P. (2000). Social categorization: Cognitions, values and groups. En C. Stangor, C. Stangor (Eds.). Stereotypes and prejudice: Essential readings (pp. 49-63). New York, NY US: Psychology Press.

Tajfel, H., \& Turner, J. C. (1979). An integrative theory of intergroup conflict. En W. G. Austin \& S. Worchel (Eds.). The social psychology of intergroup relations (pp. 33-48). Monterey, CA: Brooks/Cole.

Tajfel, H., \& Turner, J.C. (1986). The social identity theory of intergroup behavior. En Worchel, S., \& Austin, G.S. (Eds.). Psychology of intergroup relations (pp. 7-24). Chicago: Nelson-Hall.

Tajfel, H., \& Turner, J. C. (2004). The Social Identity Theory of Intergroup Behavior. En J. T. Jost, J. Sidanius, J. T. Jost, J. Sidanius (Eds.). Political psychology: Key readings (pp. 276-293). New York, NY US: Psychology Press.

Vives, J. (1984). Identidad mexicana: Un proceso con cinco siglos de duración. Psicología Iberoamericana, 2(3), 14-20.

Vignoles, V. L., Regalia, C., Manzi, C., Golledge, J., \& Scabini, E. (2006). Beyond self-esteem: Influence of multiple motives on identity construction. Journal of Personality and Social Psychology, 90 (2), 308 333. http://dx.doi.org/10.1037/0022-3514.90.2.30 